\title{
DE LOS RECURSOS DIDÁCTICOS PARA EL AULAA LOS CONTENIDOS PARA ESPACIOS VIRTUALES EDUCATIVOS CON COMPONENTES ACTIVOS Y HERRAMIENTAS DE AUTOR
}

\author{
Francisco José García Peñalvo \\ Profesor Universidad de Salamanca
}

\author{
Recibido 16-III-2004 • Aceptado 13-IV-2004
}

\begin{abstract}
Resumen: El uso de las Tecnologías de la Información y la Comunicación en los procesos educativos en general, y en la Universidad en particular, comienza a ser una realidad, al menos en apariencia y en infraestructura. Es cotidiano el uso de medios audiovisuales como apoyo a la tradicional lección magistral, así como la publicación de diversos materiales en diversos sitios web relacionados con la docencia. Sin embargo, un análisis más profundo de la situación quizás nos lleve a una realidad menos alentadora, donde el conjunto de los autores de contenidos didácticos no reúne todo el potencial humano que la Universidad podría ofrecer. En consecuencia el número de materiales didácticos se ve afectado negativamente, de modo que la calidad, organización y accesibilidad de los mismos es susceptible de importantes mejoras. Desde este artículo, apoyándonos en las experiencias desarrolladas en el Laboratorio de Diseños Educativos Multimedia y Teleeducación del Instituto de Ciencias de la Educación de la Universidad de Salamanca, se aboga por la necesidad de superar la mera creación de elementos docentes aislados y caminar hacia el establecimiento efectivo de verdaderos espacios virtuales educativos, que deberían estar soportados por componentes educativos activos y herramientas de autor para la población de dichos espacios con contenidos de calidad. En el caso de las herramientas de autor, defendemos que las interfaces autor-herramienta han de estar pensadas para facilitar el acercamiento como autores de contenidos a un personal que no tiene por qué ser experto en informática.
\end{abstract}

Palabras clave: Educación, Espacio Virtual Educativo, Herramienta de Autor, Software Educativo.

\section{Introducción}

La introducción de las Tecnologías de la Información y de la Comunicación (TIC) como instrumento y contexto de comunicación constituye un gran potencial que influye en prácticamente cualquier elemento de nuestra sociedad. Los procesos educativos universitarios no son, y no deben ser, ajenos a las tremendas posibilidades que se derivan de estas tecnologías.

Términos tales como espacios virtuales educativos, portales educativos adaptativos, formación a través de Internet (e-learning), objetos de aprendizaje... se están introduciendo rápidamente en el vocabulario de los profesionales de la educación, aunque muchos de ellos siguen sin aceptar al ordenador como una herramienta cotidiana de trabajo.

Que las TIC tienen mucho que aportar a la mejora de la calidad docente en la Universidad está fuera de toda duda, es, a nuestro juicio, un complemento (que no un sustituto) indispensable en los procesos educativos. Sin embargo, se debe ser consciente, de cara a actuar en consecuencia, de los problemas o riesgos que estas tecnologías conllevan en este momento crítico 


\begin{abstract}
At least apparently, given today's infrastructures, both IT and Communications Technologies are becoming real players in the field of educative processes at large, and this is particularly true in appearance and infrastructure. Use of audiovisual means is commonplace as a complement for standard lectures, and so is the publication of various materials in different teaching-related web sites. However, a closer look at the actual situation will perhaps take us to a less pleasant reality, in which the group of educators who produce didactic contents by no means includes all of the human potential that a University could offer. As a consequence, the number of didactic products is affected adversely, and the quality, organization and accessibility of the said products could and should be better. In this paper, which is based on the experience acquired in the Laboratorio de Diseños Educativos Multimedia y Teleeducación del Instituto de Ciencias de la Educación of University of Salamanca, we advocate the need to go beyond the mere creation of isolated teaching elements, thus advancing towards the establishment of authentic virtual teaching spaces. These should be based on active educative components and authoring tools, in such a way that the population of these spaces with quality contents could be a fact. About authoring tools, we claim that author-tool interface must be intended to facilitate and deepen their use for the wide group of content creators who are not versed in computers.
\end{abstract}

Key words: Education, Virtual Educative Spaces, Authoring Tools, Educational Software. en el que se abren camino de una forma arrolladora.

Las TIC acarrean dos problemas especialmente graves, los cuales están estrechamente relacionados (García y García, 2001). Primeramente está la falta de calidad y de organización de los materiales docentes que debe atajarse mediante el establecimiento de auténticos espacios virtuales educativos, que pongan al alcance de todos los involucrados en los procesos educativos los recursos, los servicios y las herramientas adecuadas para enfocar las TIC hacia una mejora sustancial de los procesos educativos. Por otro lado, es importante poblar de contenidos digitales dichos espacios educativos, y es aquí donde se pone de manifiesto la barrera que las nuevas tecnologías imponen a multitud de docentes, que se ven en el ostracismo tecnológico y quedan relegados en los procesos formativos actuales. Nuestra intención es recuperarlos como autores de recursos educativos para la teleformación, creando herramientas a su medida, no a la medida de los expertos en informática y comunicaciones, con interfaces que faciliten e integren, y que hayan sido pedagógicamente definidas.

Por ello, el objetivo de este artículo es presentar diversos productos de software educativo que se han desarrollado en el Laboratorio de Diseños Educativos Multimedia y Teleeducación del Instituto de Ciencias de la Educación de la Universidad de Salamanca. Estas aplicaciones software se orientan hacia la construcción de espacios virtuales formados por componentes educativos de tres tipos: i) contenidos; ii) servicios; y iii) herramientas de autor.

Además, creemos que la madurez de una organización educativa en el contexto de las TIC, puede discernirse por la forma en que ésta invierte sus mayores esfuerzos. Por ello sería factible la definición de un "modelo de madurez" que sirva como referencia para seguir la evolución del uso de las TIC en cualquier organización, de forma que cuanto más se avanzara en los 
niveles impuestos por dicho modelo, mayor sería la madurez y, por tanto, la riqueza y el beneficio obtenido con el uso de las TIC.

El resto de este artículo se organiza como sigue: en la sección dos se van a introducir algunos de los condicionantes que, según la experiencia obtenida, marcan de una forma significativa la creación de recursos, en general orientados a los espacios virtuales educativos. La tercera sección propone un modelo de madurez basado en niveles discretos que sirvan como referencia a una organización educativa para conocer cómo es su evolución con respecto a la aplicación de las TIC en el terreno educativo. En la cuarta sección se hace un repaso a diferentes aplicaciones de software educativo realizadas en el seno de nuestro grupo de investigación. Por último, la sección cinco cierra el artículo con las conclusiones del mismo.

\section{Condicionantes para la creación de recursos en los espacios virtuales educativos}

Toda sociedad humana y toda cultura se instituyen dentro de flujos de información. La cultura es un sistema funcional que opera desde la información y produce información (Mosterin, 1994). La introducción de un nuevo sistema de comunicación, no una nueva lengua o un nuevo sistema de gestos o movimientos corporales, sino la invención de un artificio comunicacional con la directa intención de expandir las propiedades del sistema de comunicación humano biológicamente dispuesto, llevará consigo la recomposición de todo un estrato del sistema de interacciones en la zona de construcción del conocimiento, precisamente el más público; esa recomposición arrastrará una reorganización de la estructura social, de las posibilidades organizacionales del tejido social y, evidentemente, una fractura generacional, porque sitúa a la generación adulta ante las nuevas formas de dependencia de información que genera el intruso.
Esta perspectiva, que es fácil de analizar en la comunicación formativa en contextos orales y lectoescritores, se pone de manifiesto en su extensión cuando se estudian las aplicaciones pedagógicas de las TIC: tecnópolis (Postman, 1993), telépolis (Echeverría, 1994), telepolitas (Echeverría, 1995).

Parafraseando el pensamiento de Castells (1998), este nuevo paradigma cultural se caracteriza por los siguientes rasgos característicos:

- La información radicaliza su carácter de materia prima; dominan las tecnologías que actúan sobre la información, frente a una información que operaba sobre el desarrollo tecnológico.

- La mediación tecnológica se interfiere en la globalidad de la actuación del Sistema Social.

- $\quad$ Se profundiza en una lógica en red de las aplicaciones, morfología reticular de los lugares de actividad que promueven, superando las limitaciones espaciales.

- Se producen cambios profundos en las categorías desde las que se piensan todos los procesos de la práctica humana. Se introducen las categorías de complejidad, como una nueva manera de considerar la diversidad, y se crean lugares de información de actividad participada.

No obstante, cuando se asume la presencia (y en muchos casos la dependencia) de este nuevo sistema de comunicación e información que nos marcan las nuevas tecnologías, debemos establecer qué condicionantes aparecen a la hora de integrar este sistema de información en nuestro entorno de trabajo, que en el caso que nos ocupa es el entorno académico o docente.

Generalmente, se suele empezar por indicar que la primera condición hace 
referencia al equipamiento. Nuestra experiencia de trabajo ha terminado por convencernos de que los primeros problemas por superar tienen más que ver con la inteligencia social que con la capacidad técnica.

Se hace imprescindible la creación de equipos para activar procesos de producción de recursos educativos actualizados. Aumentar la capacidad de la red, la velocidad de cálculo, la capacidad de almacenamiento, la sofisticación de lo que puede ser hecho, es únicamente aumentar la capacidad potencial de la calidad de acción que puede ser llevada a cabo dentro de las posibilidades del sistema técnico. El objetivo es reutilizar los recursos humanos disponibles para la reconstrucción de las funciones de formación posibles desde que se ha introducido en el contexto el nuevo sistema de comunicación. Ello requiere capacidad de integración de los conocimientos de ingeniería de telecomunicaciones e ingeniería de software, así como de la experiencia acumulada y de las habilidades relacionales de todos los involucrados.

El valor del contenido simbólico no lo construyen las TIC. Los nuevos materiales fabricados por "autores=equipos interdisciplinares” cada vez se hacen más necesarios. La volatilidad especulativa de las empresas TIC han puesto el objetivo, para algunos, en la producción de recursos de formación que pudieran invadir el mercado. El objetivo fundamental no es el económico sino el de no dilapidar recursos humanos que el nuevo contexto TIC margina, el de recuperar la capacidad de autor inhabilitada por la naturaleza misma del contexto TIC. Un objetivo de prioridad en las instituciones de formación es la creación de equipos de "autor-interdisciplinar" con la intención de recuperar la capacidad de creación en el nuevo espacio. Somos conscientes de la dificultad que entraña la creación de estos equipos y la producción de estos materiales, por haberlos promovido. Pero son imprescindibles. No debiera ser posible en el futuro una Facultad de Educación o un Centro de Formación de Profesores sin la existencia y actividad de un "Laboratorio de Diseño y Producción de Recursos de Formación" que pudieran ser aplicados tanto a la formación a distancia pura como al refuerzo de las actividades docentes tradicionales.

La producción de recursos en este momento se encuentra vinculada al empleo de las TIC, independientemente de que se produzcan para actividades de formación presencial o a distancia. Asociar las TIC a la educación a distancia es un error de bulto. La pregunta es: dentro de los objetivos socioculturales identificados y con los recursos reales valorados disponibles ¿qué puede ser hecho? Porque las TIC no son instrumentos de comunicación a distancia sino instrumentos para la captura, elaboración, almacenamiento y distribución de información.

De ahí que antes, o al mismo tiempo, de que nos planteamos cuestiones razonables de respuesta formativa a distancia, tenemos que plantearnos el empleo de recursos tecnológicos para la mejora de la calidad de formación programada. En el contexto TIC un parámetro fundamental a valorar es el nivel de competencia capitalizable para la formación que poseen los formadores y los que tienen la capacidad de decisión de la formación, porque la toma de decisiones tiene lugar dentro de un debate sobre la acción razonable.

En este orden de cosas la primera gama de decisiones políticas tiene que ver con equipamiento e infraestructura de comunicaciones en los Centros de Formación; con reorientación de las prioridades de gasto en las unidades familiares y en las demandas de consumo de las personas adultas, porque en todos los casos el acceso a la tecnología y la utilización de la misma se incorpora a los capítulos significativos de gasto personal o familiar. Mientras que en la escritura podíamos hablar de propietarios de libros, de papel o de materiales de 
escritura, aquí hay que hablar de los "Señores del Aire" (Echeverría, 1999). El soporte del sistema de comunicación tiene un elevadísimo coste que tiene que ser distribuido. Desde este punto de vista del entorno de información, como desde la economía global, vale el principio de que "nuestros pobres son más pobres", acceder al entorno TIC tiene un costo añadido. Aparece la necesidad de nuevas formas de control social de la distribución equitativa de riqueza y oportunidad, nuevos temas de debate sobre justicia social.

Desde el punto de vista de la formación, respecto a la capacidad del canal, el sistema de comunicación digitalizada constituye un gigantesco canuto vacío. La urgencia más inmediata es la de producción de contenidos de formación. Contenidos que, dada la situación actual de la zona de construcción del conocimiento creada por las TIC, todavía no tiene un mercado tan definido, claro, diversificado y reconocible como el del libro. Mientras tanto "navegación" significa posibilidad de recorridos sin rosa náutica por un caos de datos. Además, las TIC están creando herramientas de autor que permiten la construcción de contenidos formativos, pero la complejidad de manejo de éstas aleja la condición de autor de la mayor parte de los agentes de formación. Aparecen, pues, dos líneas de trabajo e investigación para equipos interdisciplinares:

1. Urge la creación de interfaces facilitadoras que permitan recuperar como autores de contenidos a los que en contexto lectoescritor están funcionando como tales en las instituciones de formación, y

2. Parece igualmente urgente identificar y organizar a los educadores hábiles e innovadores para crear equipos incentivados de producción de recursos de formación.
El valor acreditado de participación en cursos de formación tiene que ser por lo menos equivalente al trabajo de producción de recursos de formación de utilidad colectiva. Hacer depender esta producción de las industrias culturales y del mercado aumenta el impedimento para la creatividad de los actores de la formación; este impedimento ya es grande por el hecho mismo de estar implicada una tecnología y por las exigencias técnicas de las herramientas de autor. Si no evitamos que esto ocurra, la iniciativa de creación de recursos educativos cada vez estará en menos manos.

La necesidad de estos equipos interdisciplinares vuelve a subrayar la importancia del trabajo en equipo entre agentes de formación. Afrontar el problema que plantea el contexto de las TIC para la formación requiere de grandes habilidades técnicas, pero, sobre todo, requiere de grandes dosis de inteligencia social, como ya se ha recalcado.

La posibilidad de comunicación en red abierta genera nuevas posibilidades de cooperación entre equipos de profesores, tutoría de la actividad de alumnos, acceso de alumnos a información complementaria de uso colectivo... De momento, Internet se presenta como un campo abierto de relación con la información, sin tutoría y sin plan ni diseño curricular, salvo en los denominados centros virtuales de formación. Creemos que un objetivo político es el de recuperar en el nuevo contexto el concepto de institución de formación, el del proyecto colectivo de formación que adquiere cuerpo social, en este caso, con soporte de información. La vía puede ser la de la creación de intranets, o redes corporativas, dentro del modelo, a menor escala de las instituciones financieras. Ello permitiría recuperar el concepto y la iniciativa de movimientos de renovación pedagógica en el contexto de las TIC. En relación con la aparición de la red como canal de comunicación, y de una forma más concreta, se podría hablar de: 
- Crear nuevos sistemas de comunicación entre el profesorado, lo que ofrece un nuevo sentido al concepto de Equipos Docentes y de recursos de formación disponibles, tanto en la fase de elaboración colectiva como de empleo por los estudiantes.

- Redefinir el concepto de formación permanente del profesorado mediante comunidades colaborativas en red, lo que favorecen los servicios de foros de discusión, tanto síncronos como asíncronos, así como otras plataformas de cooperación que podrían diseñarse para este fin.

- Replantear el concepto de agente educativo del sistema, extendiéndolo a los profesionales de la formación que reciban la responsabilidad de alimentar las plataformas de ayuda y de recursos colectivos a distancia. Trabajar para los mismos usuarios, del mismo nivel de enseñanza, con el mismo diseño curricular pero en régimen de teletrabajo.

- Publicitar a bajo costo, haciendo uso de las nuevas formas que ofrece la Red, la actividad ejemplar de profesores en sus aulas y la producción de materiales innovadores. Este campo estaba antes limitado por los costos de edición.

La Pedagogía en el contexto de las TIC debe crear "Laboratorios de Diseños Educativos Multimedia y Teleeducación”, con el objetivo del diseño, construcción y producción de componentes para espacios virtuales de formación (Echeverría, 2000; García y García, 2001). La ingeniería telemática y tecnología informática aplicable están alejando la posibilidad de que los pedagogos puedan seguir siendo creativos. Esa posibilidad puede volver a encontrarse en equipos multidisciplinares que reflexionan sobre las múltiples necesidades de formación en la zona de construcción del conocimiento y en las versátiles maneras de interpretarlas.

La fractura general más evidente y su prueba más fehaciente es la que se está produciendo en este momento entre los niños que llegan a las aulas, que son usuarios de los ordenadores en sus casas, y los profesores en los centros, que pueden haber perdido ya la ilusión de seguir siendo actores, sin casi haber probado a coger entre los dedos el "nuevo bolígrafo".

Después de todo lo dicho, el problema fundamental de una política de formación, vuelve a ser la formación del profesorado en el nuevo contexto de información: la formación de formadores. La prioridad no está en formarlos en empleo de sofisticadas herramientas informáticas. El plan de formación de los formadores debe corresponderse con el proyecto de acción en el que han de verse implicados. También la formación de los formadores debe ser diversificada, personalizada, permanente y situarse en un contexto de aprovechamiento de las TIC, dentro del cual se les pide que sean agencias de formación. Debemos recuperar iniciativas locales-regionales de renovación pedagógica, tendrán que ser interdisciplinares y asociadas a proyectos de formación en el medio plazo.

\section{Modelo de madurez}

La incorporación significativa de las TIC en el proceso educativo propio de una determinada organización o entidad no es algo que de forma instantánea llegue a todos los niveles de dicha organización. Más bien se puede asegurar que la asimilación de estas tecnologías es un proceso incremental, donde cada incremento supone un mayor grado de madurez para la organización.

Con el objetivo de conocer la evolución de una organización con respecto a la implantación, asimilación y uso de las TIC, puede ser interesante definir un modelo de madurez que otorgue dicho conocimiento. 
Nos ha parecido interesante utilizar el nombre de modelo de madurez siguiendo la metáfora, ya utilizada en el mundo del software, para significar una correlación entre el uso útil y maduro de las nuevas tecnologías y el beneficio que éstas deben aportar a la globalidad de la organización donde este modelo se aplica.

En nuestro grupo se han esbozado los principios de un modelo que, de forma semejante a otros modelos (CMM - Capability Maturity Model (Paulk et al., 1993), por ejemplo), define una serie de niveles discretos que permiten conocer tanto el nivel de madurez de la organización con respecto a las TIC, como los pasos a seguir para alcanzar un nivel mayor de madurez. En la Figura 1 se recogen las bases de este modelo de madurez.

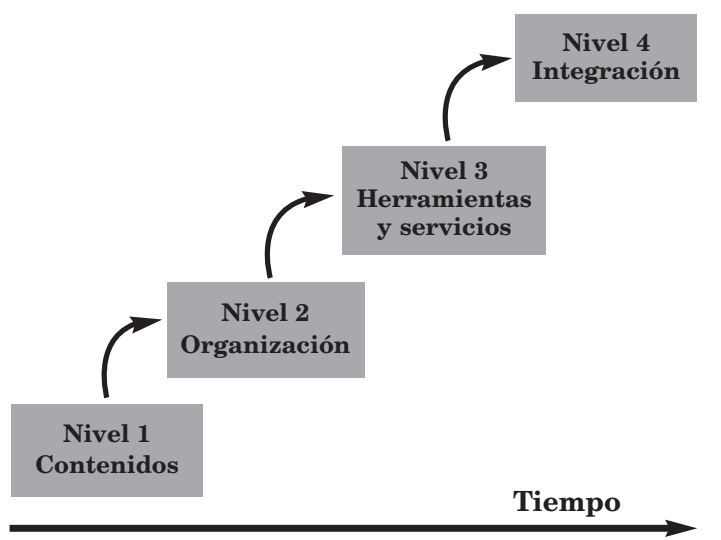

Figura 1. Esquema del modelo de madurez.

El primer nivel, o nivel de contenidos, representa el menor grado de madurez. La organización facilita medios técnicos a los docentes, y éstos sólo se preocupan por la creación y uso de los contenidos. En este nivel tienen cabida actividades como la creación de apuntes, transparencias, programas interactivos, demostraciones..., pero todo este proceso creativo tiene lugar de una forma ad-hoc, no organizada ni canalizada para ser efectiva en el ámbito de la organización. Los esfuerzos son personales y, normalmente, dependen de las habilidades que cada docente (o grupo de docentes) tenga con unas determinadas herramientas de autor. Con frecuencia estos contenidos no están preparados para entrar a formar parte de un espacio virtual educativo, salvo para su descarga completa, esto es, como si se tratara de un libro o de unos apuntes que en lugar de adquirirlos en papel se consiguen en un formato digital.

El segundo nivel, nivel de organización, establece una preocupación no tanto en la creación de los contenidos, sino en los mecanismos existentes para facilitar la organización y el acceso a los contenidos existentes. En este nivel aparece un sentimiento institucional en la organización y se pretende canalizar el acceso a los recursos creados o seleccionados por sus docentes. Aparece el concepto de portal o plataforma educativa virtual que combina, en un primer estadio, las facilidades de repositorio o almacén de información con un conjunto de servicios básicos para la gestión y el acceso a los recursos de contenido. Habitualmente la oferta de servicios se ve incrementada con un conjunto de elementos de comunicación que buscan poner en contacto los roles de docente y de discente. Esta oferta de servicios incluye, típicamente, mecanismos de comunicación asíncrona, como el correo electrónico o los grupos de noticias, y mecanismos de comunicación síncrona, como el chat.

El tercer nivel es un nivel funcional, en el que predomina la oferta de servicios de mayor nivel, accesibles a través de las plataformas establecidas en el nivel de organización $\mathrm{u}$ otras construidas de forma personalizada y que se comunican o envuelven a las ya existentes. Uno de los servicios que aparecen ligados a este nivel de madurez es el de soporte a la autoría de contenidos mediante herramientas de autor construidas, destinadas y pensadas para los autores de contenidos, y que dan el soporte necesario para lograr los objetivos de los niveles uno y dos de la forma más 
automática posible. En estas herramientas de autor se debe facilitar y soportar, en la mayor medida de lo posible, el trabajo cooperativo entre autores que pueden estar geográficamente dispersos.

Finalmente, el último nivel de madurez, o nivel de integración, busca como objetivo la integración en un espacio virtual educativo de los contenidos, la plataforma en que se organizan y los servicios. Este espacio virtual debe reflejar el flujo de trabajo del proceso educativo que representa y establece la forma adecuada de llegar, compartir y reutilizar los contenidos docentes. Esta integración debe hacerse a dos niveles, usuario y contenidos. El nivel de usuario destaca aspectos tales como la interacción sistema-usuario, presentando interfaces centradas en el usuario que potencien la usabilidad del sistema para todos sus usuarios, la adaptatividad a las preferencias y conocimiento de los usuarios, sobre la base de modelos de usuario y de un cierto nivel de inteligencia, o la gestión del conocimiento, que permita un soporte adecuado a la toma de decisiones. Por su parte, el nivel de contenidos debe construirse sobre una base semántica estandarizada que dé lugar a trabajar con Objetos de Aprendizaje, que con un recubrimiento semántico envuelven a los contenidos tradicionales, al nivel que de granularidad que se desee, y que facilitan la interoperabilidad y reuso de los contenidos en diferentes plataformas, de una forma transparente y automática, lo que nos acercaría al concepto defendido por la Web Semántica (Berners-Lee et al., 2001).

Obviamente un sistema de cuatro niveles discretos, como el que aquí se presenta, no ofrece respuestas a todos los interrogantes que pueden aparecer en la aplicación de las TIC en el proceso educativo. De la descripción realizada se puede intuir cómo cambiar de nivel, pero nada se ha dicho sobre el camino a seguir dentro de un nivel determinado. En este sentido, y sin entrar en mayores detalles, nosotros pensamos que dentro de cada nivel se establecen varias iteraciones incrementales que van consolidando la madurez de la organización y que son las responsables a su fin de conseguir el salto cualitativo y de madurez que supone el cambio de nivel en el modelo propuesto.

En nuestro caso concreto la organización sería el Laboratorio de Diseños Educativos Multimedia y Teleeducación del Instituto de Ciencias de la Educación de la Universidad de Salamanca, y consideramos que nos encontramos en un nivel tres de madurez, aunque con un largo camino por recorrer dentro de este nivel antes de llegar a un nivel óptimo de integración.

Durante la fase orientada a los contenidos, el grupo de trabajo desarrolló diversos productos educativos en diversos campos como las comunicaciones, las matemáticas o la economía entre otros, siendo productos con una importante carga multimedia y distribuidos en soporte CD-ROM.

En la fase de organización se pueden distinguir diversos esfuerzos orientados a la construcción de portales temáticos en Internet donde alojar contenidos, como por ejemplo TutorTE orientado a personas de la tercera edad (Therón et al., 2002), así como en la adopción, en un ámbito institucional dentro de la Universidad de Salamanca, de una plataforma global (EUDORED) donde organizar contenidos digitales para ofertar cursos no presenciales.

Ya dentro del tercer nivel, y gracias a una relación sinérgica dentro del Laboratorio entre personal interdisciplinario proveniente fundamentalmente de las áreas de la Pedagogía y de la Ingeniería Informática, se han desarrollado diversos componentes pedagógicos activos y herramientas de autor, a la par que se tienen en desarrollo otros muchos más, en los que ya se empiezan a utilizar especificaciones o estándares sobre tecnologías de aprendizajes para almacenar semánticamente los contenidos generados, concretamente se 
ha hecho uso de EML (Education Modelling Language) (Koper, 2001) y de las especificaciones IMS (2003).

En la siguiente sección se va a hacer un recorrido por el trabajo realizado en este apartado, destacando alguno de los componentes desarrollados.

\section{Componentes activos y herramientas de autor}

Nuestro trabajo refleja la evolución en este proceso de madurez, de forma que se ha pasado de poner el énfasis de nuestros componentes en el desarrollo de contenidos interactivos independientes, a la elaboración de herramientas de autor que faciliten la labor de los profesores a la hora de crear componentes educativos, recuperándolos como "autores" para una forma de docencia cada vez más relacionada con las TIC.

Todo el trabajo va encaminado hacia el establecimiento de un entorno de e-formación que pretende ser un portal educativo configurable a las necesidades de las actividades que en él vayan a tener cabida, cuidando siempre dos elementos decisivos: la accesibilidad y la mejora del aprendizaje.

A través de los componentes desarrollados, el proceso de aprendizaje se abre a todo tipo de alumnos, desde niños a adultos de avanzada edad. La optimización del aprendizaje se logra al flexibilizar el proceso, potenciando un sistema asíncrono de estudio, salvo para actividades concretas, normalmente dirigidas a un público especializado, concreto y de avanzados conocimientos.

Actualmente existen numerosos componentes desarrollados, que han sido ampliamente probados en diferentes experiencias educativas, y otros muchos se encuentran en desarrollo. Los componentes desarrollados evolucionan hacia nuevas versiones, buscando la mejora continua de sus prestaciones y, por tanto, del espacio virtual educativo que conforman.
Los componentes desarrollados pueden clasificarse siguiendo diferentes patrones de categorización, sin embargo, para los fines de este artículo se van a dividir en dos grupos: componentes de servicio educativo (propios del nivel 2 del modelo madurez presentado) y componentes de autor (propios del nivel 3 de dicho modelo de madurez).

Los componentes de servicio educativo están destinados a dar soporte a actividades docentes concretas, en las que la colaboración es una característica fundamental. Entre los componentes desarrollados, cabe citar a: el seminario virtual (Moreno et al., 2000b) -que permite la realización de seminarios, cuyos participantes están geográficamente distantes; el tutor de ortografía (GUIO v1) (Rodríguez et al., 2002)- destinado a que los niños aprendan y corrijan sus problemas con las reglas de ortografía del español; o el gestor para seguimiento de tesis (Moreno et al., 2000a) - que presenta un entorno para el seguimiento de trabajos académicos por parte de un tutor (tesis, tesinas o proyectos de final de carrera).

Los componentes de autor están orientados a que el docente cree y componga materiales didácticos de calidad que incorporará de alguna manera al espacio virtual. Entre otros varios componentes desarrollados, cabe citar a: el gestor de actividades -destinado a la creación de unidades docentes, que incluyen problemas y su resolución; el compositor de hipertextos (HyCo v2) (García et al., 2003; García y García, 2004), herramienta destinada a crear documentos hipermedia con estructura jerárquica, los cuales quedan almacenados en formato semántico basado en XML (Bray et al., 2004) y que pueden ser exportados según las especificaciones EML (Koper, 2001) o IMS (2003); o el generador de guías académicas (Moreno et al., 2000a), que auxilia al docente en la creación de las guías de las asignaturas que imparte.

A continuación se van a presentar de una forma más detallada, el seminario 
virtual, el tutor de ortografía (GUIO) y el compositor de hipertextos ( $\mathrm{HyCo}$ ).

\subsection{El seminario virtual}

Este componente permite la organización, coordinación y realización de seminarios virtuales entre usuarios conectados a Internet con unas prestaciones adecuadas para el correcto devenir del mismo, así como para un intercambio de documentación sobre el tema abordado.

La aplicación debe facilitar el trabajo colaborativo de los participantes en el seminario, usuarios que pueden estar geográficamente distantes y no tienen por que ser expertos en informática, así como gestionar la correcta fluidez de la información para asegurar que ésta pueda ser asimilada, sin llegar a desbordar.

Los objetivos funcionales del seminario virtual fueron diseñados desde un prisma pedagógico y fundidos en la interfaz de usuario para maximizar la usabilidad de la aplicación, siendo el conjunto de metáforas empleadas para materializarlos uno de los puntos más interesantes y destacables de este componente educativo.

Con el fin de cumplir con los objetivos propuestos se ha dotado a la herramienta de las siguientes facilidades:

- Zona de diálogo e identificación de los participantes. Esta zona se corresponde con la estructura de un chat clásico.

- Moderación del seminario. Existe la figura del moderador del seminario que es quien otorga la palabra a los participantes que lo soliciten.

- Zona de carga y descarga de documentos. Esta zona denominada corcho, permite el intercambio de información organizada en ficheros entre todos los participantes del seminario.
- $\quad$ Pizarra. Esta zona constituye el núcleo de la aplicación siendo el lugar donde se desarrolla el seminario a semejanza de cómo se haría con una pizarra clásica.

- Almacenamiento de los seminarios. Facilidad que permite visualizar los mismos con posterioridad y que convierte un servicio intrínsecamente síncrono en un servicio asíncrono a voluntad de los participantes.

La organización de estos apartados en la interfaz gráfica se puede apreciar en la Figura 2. En la parte superior se sitúa una barra de herramientas que contiene un icono para ver la ayuda en formato HTML, un botón para conectar y desconectar y una barra de estado donde se muestran algunos mensajes informativos. Inmediatamente debajo a la derecha aparece la zona de identificación de participantes y de diálogo, mientras que en la zona izquierda está la zona de carga y descarga de documentos, el corcho, y la pizarra.

La zona de pizarra constituye la parte central de la aplicación. Permite emular a una pizarra clásica, aunque más versátil pues es posible visualizar en ella cualquiera de los ficheros depositados en el corcho, a semejanza de un proyector de transparencias. Esta utilidad permite apoyar las explicaciones en dibujos, fórmulas, esquemas, diagramas... Adicionalmente, haciendo uso de los elementos presentes en la barra de herramientas de la pizarra, que representan metáforas ligadas al proceso educativo como la tradicional tiza o el moderno puntero láser, el ponente puede hacer hincapié en aquellas partes de la exposición que considere necesario.

\subsection{El tutor de ortografía (GUIO v1)}

La enseñanza de la ortografía es uno de los graves problemas al que ha de 
enfrentarse el profesorado de Educación Primaria, sin que en la actualidad, se haya conseguido un recurso de calidad que la posibilite en toda su complejidad.

Como medio de combatir estas vicisitudes, se ha desarrollado un software educativo destinado a niños y niñas con edades comprendidas entre los 8 y los 13 años (lo que correspondería en el ámbito educativo al segundo y tercer ciclo de Educación Primaria). Este software no se limita a ser un mero transmisor de reglas ortográficas, aspecto de alguna forma secundario. Por el contrario, partimos del aprendizaje de un vocabulario con demostrada dificultad ortográfica como eje alrededor del que girará el proceso de instrucción.

Otro criterio en el que también se fundamenta todo el diseño de este software educativo es el componente lúdico. Se pretende que los pequeños usuarios del mismo se acerquen al aprendizaje: a través del juego, de una interfaz cuyas ilustraciones respondan a sus gustos, de la elección de temáticas atractivas en las que se introducen los contenidos a aprender; todo a fin de asegurar la incentivación y despertar sus intereses hacia una interacción efectiva que asegure la asimilación de los aspectos a aprender.

El resultado ha sido GUIO v1.0, un entorno para la evaluación y la enseñanza de la ortografía en Educación Primaria.

Este componente educativo consiste, a grandes rasgos, en una aplicación multimedia, mediante la cual el niño adquiere y/o refuerza sus conocimientos sobre la ortografía a través de juegos y motivaciones positivas, ejerciendo una actividad de aprendizaje que de otra forma pueda llegar a resultar aburrida y tediosa.

Dada las características de los usuarios de esta aplicación se decidió incluir la metáfora del asistente en la interfaz de la herramienta, pero había que hacerlo de forma que los alumnos se identificasen con ellos. Así, se han creado cuatro protagonistas de la acción que se desarrolla en las 
Figura 3. Protagonistas del tutor de ortografía.

lecciones, que son cuatro niños (dos niños, Pablo y Nicolás, y dos niñas, Raquel y Dorita) cada uno de los cuales representa un carácter o un tipo de niño diferente. Además de los niños se cuenta con una mascota que fue bautizada como Guio. Todos ellos se pueden apreciar en la Figura 3.

A la hora de seleccionar los elementos que sirven de evaluación y seguimiento del alumno se pensó en aquellas actividades y juegos que permitieran divertir y a la vez mantener la atención durante su realización, de manera que, como resultado, se obtuviera un aprendizaje satisfactorio. Las actividades elegidas son:
Crucigramas, Autodefinido, Sopa de Letras, Fuga de Letras, Adivinanzas y Letras Desordenadas. En la Figura 4 se presenta una de estas actividades, la Sopa de Letras.

Con la elección de actividades de este tipo, se pretende que el alumno se habitúe a ver palabras con cierta dificultad ortográfica y además lo haga de forma lúdica, de manera que a la vez que está jugando visualiza la palabra y su estructura. Esto supone la memorización de dichos elementos y resultará más efectiva debida a la continua reiteración de mismas palabras en diferentes actividades. 


\subsection{El Compositor de Hipertextos (HyCo v2)}

Una herramienta de autor para ambientes hipermedia es un entorno en el que los autores pueden crear sus documentos hipermedia para diferentes dominios de aplicación. HyCo es una herramienta de autor que se centra en el dominio de la educación, por lo que su cometido es la creación de recursos hipermedia educativos, que serán creados con independencia del formato que se quiera utilizar para su difusión final en espacios virtuales educativos, ya sea mediante un CD-ROM o a través de la Web.

El esfuerzo desarrollado está justificado por la dificultad que encuentran un gran número de docentes en crear materiales hipermedia, principalmente por su desconocimiento de las complejas herramientas de autor que se utilizan para estos menesteres, más pensadas para profesionales de la Informática que para los expertos en otras materias. Así, mediante una interfaz sencilla y amigable y unas metáforas de interacción adecuadas, se consigue que cualquier docente vuelque $\mathrm{su}$ experiencia y conocimiento en materiales adecuados para estos espacios virtuales, con independencia de sus conocimientos informáticos.

Este componente es a la vez una herramienta de autor (o administrador) y de usuario (o lector), de manera que se encapsula toda la complejidad del manejo de las herramientas actuales en las facilidades que el autor necesita, y ofrezca como resultado un producto didáctico (Aedo y Díaz, 2000), hipermedia distribuible en diferentes formatos.

Básicamente la herramienta reproduce el proceso que seguiría un autor para crear un recurso educativo lineal, pero canalizándolo, a la vez que organizándolo, a través de la metáfora de índice de contenidos, y añadiéndole las facilidades para la inclusión de elementos multimedia (imágenes, vídeos, sonidos...) así como de hipervínculos.
En los siguientes subapartados se va a desgranar el proceso de creación de un documento hipermedia con esta herramienta y se van a presentar algunas de las facilidades que incluye esta herramienta de autor.

\subsubsection{Creación de un recurso docente}

Dentro del modo autor se puede crear un nuevo libro o abrir uno existente para su modificación. Cuando un autor se dispone a crear un texto, educativo o no, es recomendable, por no decir imprescindible, realizar una organización de los contenidos, en pro de la claridad y calidad del resultado final.

Una forma comúnmente aceptada de llevar a cabo esta organización es la realización previa de un índice de contenidos, sin límite teórico de niveles de anidamiento (aunque si práctico en aras de la claridad), de forma que se obtenga una estructura jerárquica que dirija en nuestro proceso creativo, el cual consistirá en ir asociando contenidos a cada entrada del índice; índice que por otra parte puede sufrir variaciones según se van dando forma a los contenidos, insertando, eliminando o cambiando entradas del mismo.

De esta manera, el compositor de hipertextos reproduce fielmente el proceso anteriormente explicado. La interfaz de trabajo presenta dos zonas diferenciadas y destacadas del resto, la zona de índice y la zona de trabajo, como se puede apreciar en la Figura 5. Cada entrada del índice da lugar a una unidad temática o lexia que puede contener texto, material multimedia y enlaces a otras unidades o documentos.

Esta organización, además de dar lugar a una metáfora de trabajo fácilmente asimilable por cualquiera que haya escrito antes algún tipo de documento jerárquico, permite una traducción directa a un hiperdocumento bien organizado, sin más que tener en cuenta que cada lexia se corresponde con un nodo del hiperdocumento.

La equivalencia lexia-nodo es la base para el éxito del proceso de creación porque 
Figura 5. Interfaz de trabajo: Zona de índice + zona de trabajo.

el nodo, al igual que la lexia para el autor, es una unidad de información en la que una serie de contenidos de diversa índole se combinan para transmitir una idea o concepto. El nodo es una unidad de visualización que facilitará la labor de los navegadores para mostrar el hiperdocumento y navegar a través de él. Además, esta equivalencia cumple una máxima de calidad de todo hiperdocumento, los nodos deben ser entidades autocontenidas para evitar que un concepto se divida en múltiples fragmentos, de forma que a los lectores se les facilite su reconocimiento.

El índice del documento sirve como navegador para desplazarse a las diferentes lexias de que se compone un hiperdocumento, de manera que en el modo de autor, permite acceder a la lexia en la que se quiere trabajar, o bien, mediante las opciones del índice, hacer las modificaciones al índice que el autor estime oportuno (ver parte izquierda de la Figura 5), mientras que en el modo de lector, junto a los hipervínculos, representa la forma de navegar por el hiperdocumento.

\subsubsection{Manejo de elementos multimedia: Las galerías}

Un hiperdocumento complementa al texto con información de diversa índole. Un problema para el autor es mantener organizada esta información, de cara a su posible publicación en un medio ajeno a la propia herramienta de autor. 


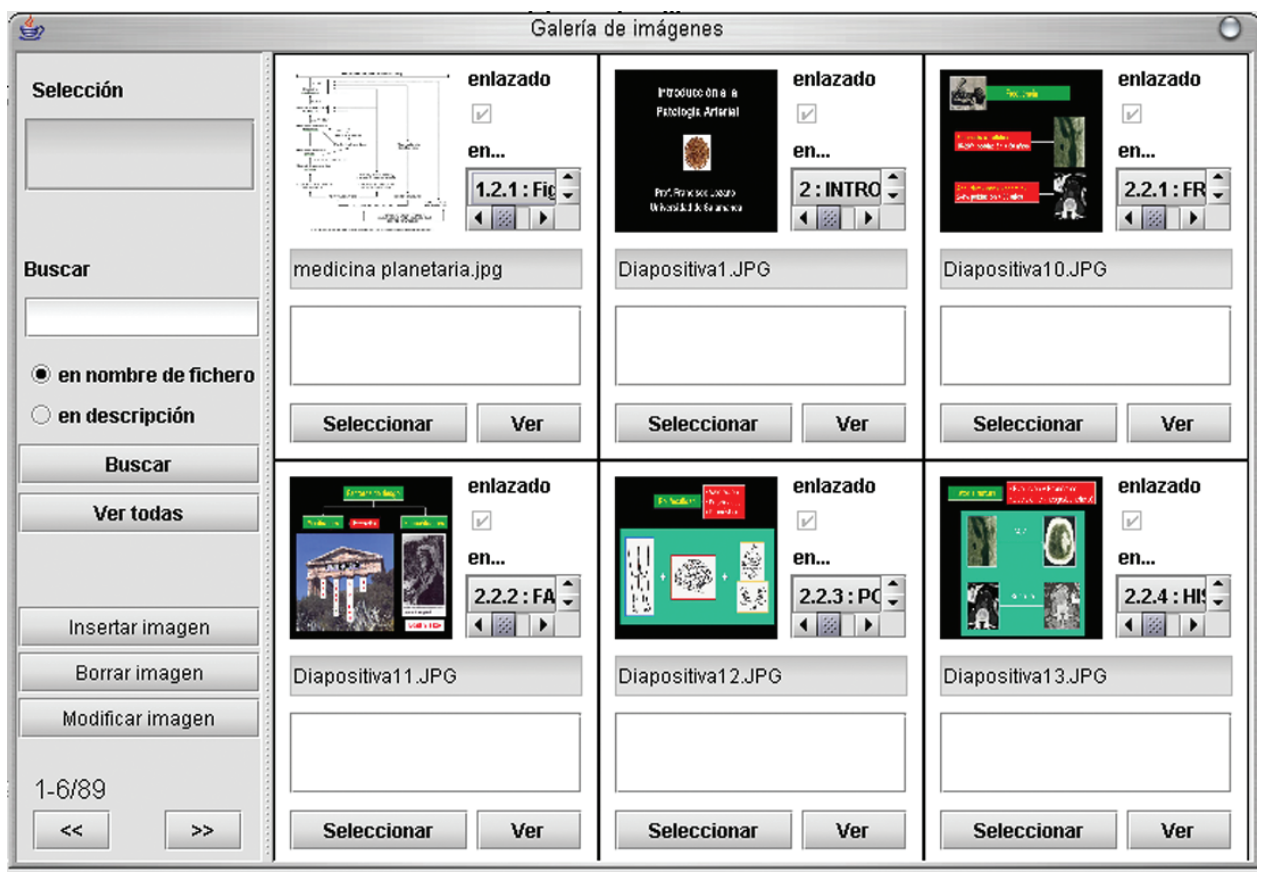

Figura 6. Galería de Imágenes.

En este sentido el compositor de hipertextos mantiene un repositorio de información vinculado a cada documento que se cree con la herramienta, de forma que se soluciona en gran parte la localización de este tipo de recursos. Pero además, para su gestión por parte del autor, se incorpora una serie de interfaces, basadas en las metáforas de visor y formulario, que reciben el nombre de galerías.

Una galería es un gestor de toda la información, clasificada por tipos, que el usuario está insertando en su documento, y que es almacenada en el repositorio del documento de forma totalmente transparente al usuario. Concretamente la herramienta cuenta con cuatro galerías: Galería multimedia, Galería de estilos, Galería de citas y Galería de salidas.

La Galería Multimedia proporciona el control sobre todos los elementos multimedia que se tengan insertados en el documento, con la posibilidad de previsualización.
Se distinguen tres tipos de Galerías Multimedia, la Galería de Imágenes, la Galería de Vídeos y la Galería de Sonidos. La forma de trabajo con las diferentes galerías multimedia es tan simple como seleccionar el texto a enlazar y elegir la opción de insertar una imagen, sonido o vídeo. En ese momento aparece la galería que corresponda en la cual se han insertado previamente los elementos necesarios, se selecciona el deseado y se enlaza. En la Figura 6 se muestra una captura de la Galería de Imágenes.

A través de la Galería de Estilos se puede cambiar la fuente y el tamaño que se utiliza en el editor.

Uno de los puntos clave en un recurso docente es la bibliografía que éste incluye. Siempre es tedioso realizar un mantenimiento de las referencias que se quieren incluir en los recursos que se crean. Para facilitar esta labor se ha incorporado la Galería de Citas, a través de la cual se realiza un tratamiento bastante avanzado de 
la bibliografía del documento. Para crear una nueva fuente bibliográfica se dispone un asistente que en cuatro pasos obtiene los datos necesarios sobre la fuente a introducir.

Por último, la Galería de Salidas conforma una de las galerías más interesantes y una gran funcionalidad de la aplicación. Mediante esta galería se permite obtener el recurso docente en diferentes formatos. En esta versión se ha conseguido proporcionar salidas a los formatos clásicos HTML, RTF, PDF, PS, SVG y TXT, o bien en formatos semánticos XML, EML o IMS.

\section{Conclusiones}

En este artículo se han expuesto una serie de reflexiones que el autor ha realizado basándose en su experiencia en el desarrollo de herramientas de autor y de utilidad, así como de contenidos formativos, todos ellos aplicados a los espacios virtuales educativos, dentro del Laboratorio de Diseños Educativos Multimedia y Teleeducación del Instituto de Ciencias de la Educación de la Universidad de Salamanca.

En primer lugar se ha hecho un recorrido por los diversos condicionantes que se deben tener en cuenta a la hora de elaborar material docente, donde la inteligencia social para la constitución de equipos interdisciplinares es un aspecto lo suficientemente relevante, como puede serlo el estar al tanto de las últimas tendencias tecnológicas.

Una de las mayores preocupaciones de nuestro grupo de trabajo es la barrera que las TIC imponen a multitud de docentes, que se ven en el ostracismo tecnológico y quedan relegados en los procesos formativos actuales. Nuestra intención es recuperarlos como autores de recursos educativos para la teleformación, creando herramientas a su medida, no a la medida de los expertos en informática y comunicaciones, con interfaces facilitadoras e integradoras, pedagógicamente diseñadas, como se puede haber apreciado en los ejemplos comentados a lo largo de este artículo.

Además, y como conclusión global se ha querido poner de manifiesto, y corroborar con nuestra experiencia concreta, como cualquier institución de carácter docente sufre un proceso de madurez, en el que va desplazando su centro de atención y, por tanto, el grueso de sus esfuerzos, de la mera creación de contenidos docentes dispersos, al establecimiento de un flujo de trabajo que canaliza dichos contenidos desde su creación, utilizando herramientas de autor adecuadas, hasta su presentación y uso, a través de las interfaces de servicio adecuadas.

\section{Agradecimientos}

Deseamos manifestar nuestro a agradecimiento al grupo AWEG -Adaptive Web Engineering Group- de la Universidad de Salamanca por sus contribuciones e ideas en el desarrollo de este trabajo.

Este trabajo ha sido parcialmente subvencionado por la Junta de Castilla y León y la Unión Europea a través del Fondo Social Europeo mediante el proyecto de investigación SA017/02 y por la Unión Europea mediante el proyecto ODISEAME (Open distance Interuniversity Synergies between Europe, Africa and Middle East), ref. EUMEDIS B7-4100/2000/2165-79 P546.

\section{Referencias bibliográficas}

Aedo, I. y Díaz, P. "Diseño de libros electrónicos educativos". Actas de las I Jornadas Interacción'2000, Granada, España. 2000.

Berners-Lee, T., Hendler, J. y Lassila, O. "The semantic web". Scientific American. 2001. 
Bray, T., Paoli, J., Sperberg-MacQueen, C. M., Maler, E. y Yergeau, F. (Eds.). "Extensible Markup Language (XML) 1.0 (Third Edition)". World Wide Web Consortium.

http://www.w3.org/TR/2004/ RECxml-20040204. 2004.

Castells, M. La societé en réseaux. L'ère de l'information. Paris: Fayard. 1998.

Echeverría, J. Telépolis. Barcelona: Destino. 1994.

Echeverría, J. Telepolitas domésticos. Barcelona: Anagrama. 1995.

Echeverría, J. Los señores del aire: Telépolis y el tercer entorno. Barcelona: Destino. 1999.

Echeverría, J. Un mundo virtual. Barcelona: Circulo Cuadrado. Colección Debolsillo. 2000 .

García, F. J., Carabias, J., García, J. y Berlanga, A. "HYCO - An authoring tool for semantic educational resources". Proceedings of the Second International Conference on Multimedia and Information \& Communication Technologies in Education (m-ICTE 2003). December 3-6th 2003, Badajoz (Spain).

García, F. J., García, J. "Los espacios virtuales educativos en el ámbito de Internet: Un refuerzo a la formación tradicional”. Teoría de la Educación. Educación y Cultura en la Sociedad de la Información, Vol. 3. España. http://www3.usal.es/teoriaeducacion/ rev_numero_03/n3_art_garcia-garcia. htm 2001.
García, F. J., García, J. "Educational hypermedia resources facilitator". Computers \& Education. In press. 2004.

IMS. IMS learning resource metadata specification.

http://www.imsglobal.org/metadata/ index.cfm. 2003.

Koper, R. "Modelling units of study from a pedagogical perspective. The pedagogical meta-model behind EML".

http://eml.ou.nl/introduction/docs/ ped-metamodel.pdf 2001.

Moreno, Á. Mª ., García, F. J., García, J. y Alonso, L. "Componentes software para entornos virutales de educación". Actas de las Jornadas UNED-2000. Conocimiento, Método y Tecnologías en la Educación a Distancia. Palencia, España. 2000a.

Moreno, Á. Maㅡ, García, F. J., García, J. y Gutiérrez, A. "Seminario Virtual: Llevando los espacios virtuales educativos a la práctica". Actas del $2^{\circ}$ Simposio Internacional de Informática Educativa-SIIE'2000. Puertollano-Ciudad Real, España. 2000b.

Mosterin, J. Filosofía de la cultura. Madrid: Alianza. 1994.

Paulk, M. C., Curtis, B., Chrissis, M. B. y Weber, C. V. "Capability maturity model, version 1.1". IEEE Software, Vol. 10, No 4, pp.18-27. EEUU. 1993.

Postman, N. Technopoly: The surrender of culture to technology. New York: Vintage. 1993. 
Rodríguez, R., Hernández, A., Lecuona, $M^{\underline{a}}$ del Pino, García, F. J., Moreno, Á. M ${ }^{\mathrm{a}}$ y Gil, A. B. "Diseño y desarrollo de una aplicación para la enseñanza de ortografía a niños de primaria: GUIO v1.0". Actas del III Congreso Internacional de Interacción Persona - Ordenador - Interacción 2002. Leganés, Madrid, España. 2002.
Therón, R., García, F. J., Moreno, Á. Mª , Gil, A. B., Curto, B., Martín, A. V. y García, J. "Servicios integrados para personas mayores". Minusval. Número Especial, pp. 40-43. Ministerio de Trabajo y Asuntos Sociales. Secretaría General de Asuntos Sociales IMSERSO. España. 2002 . 